\title{
Functional importance of the long axis dynamics of the human left ventricle
}

Christopher J H Jones, Luciano Raposo, Derek G Gibson

\section{Abstract}

The role of longitudinally and circumferentially oriented fibres in left ventricular wall motion was examined by digitising echocardiograms of the mitral ring (whose motion reflects long axis change) and of the standard minor axis in 36 healthy individuals, 36 patients with coronary artery disease, 16 with left ventricular hypertrophy, 44 with mitral valve disease ( 24 of whom had undergone mitral valve replacement). In the controls long axis shortening significantly preceded minor axis shortening (mean (1 SD) difference $25(40) \mathrm{ms}$ ) so that the minor axis increased more during isovolumic contraction $(0.25 v 0.09 \mathrm{~cm})$, indicating that the left ventricle became more spherical. Changes in the long and short axes were synchronous at end ejection and in early diastole in the controls. Epicardial excursion preceded endocardial excursion by 50 (20) $\mathrm{ms}$ at its peak. These time relations were consistently disturbed in all patient groups, irrespective of the extent of fractional shortening of the minor axis. The onset of long axis shortening was delayed, and this was often associated with premature shortening of the minor axis, the normal spherical shape change during isovolumic contraction was lost, and peak epicardial and endocardial changes became more synchronous. In patients with coronary disease these changes are the expected consequence of ischaemic injury to longitudinally orientated subendocardial fibres. In left ventricular hypertrophy their presence consistently showed systolic dysfunction when orthodox measures were still normal. They were more pronounced after mitral valve replacement when the papillary muscles had been sectioned; long axis shortening was reduced during systole and prolonged into early diastole, while normal shortening of the minor axis was maintained only by abnormal epicardial excursion.

Relations between long and short axis motion in healthy individuals are characteristic, and their loss is an early index of systolic ventricular disease. These disturbances precede changes in orthodox measures such as fractional shortening or peak velocity of circumferential fibre shortening.
Anatomical studies ${ }^{12}$ have shown longitudinal as well as circumferential fibres with a continuous variation in fibre angle across the left ventricular wall. The function of these longitudinal fibres has not been extensively studied. Because effective ventricular function during ejection and filling is likely to depend upon the coordinated action of all myocardial layers, we set out to study the timing and extent of changes in the long axis, comparing them with those of the minor axis in healthy controls and those with left ventricular disease. In addition, we investigated patients after mitral valve replacement where a component of the longitudinal fibres (the papillary muscles) had been sectioned.

\section{Patients and methods}

CONTROLS AND PATIENTS

Cross sectionally guided $M$ mode echocardiograms were recorded and analysed in 36 patients with coronary artery disease, 16 with left ventricular hypertrophy, 44 patients with mitral valve disease (10 with rheumatic mitral stenosis before surgical treatment, 24 after mitral valve replacement, and 10 after mitral valve repair), and 36 healthy individuals aged 51 (12) years without clinical, cardiographic, or radiographic evidence of heart disease and with normal amplitude and timing of endocardial motion and normal mitral valve leaflet motion on standard short axis $M$ mode echocardiography. Patients with coronary artery disease had presumed angina and were those referred for routine diagnostic angiography. This showed single vessel disease in five, double vessel disease in ten, and triple vessel disease in 21 . Cavity size and fractional shortening on $M$ mode echocardiography were normal in 22. Fractional shortening was $<23 \%$ (the lower $95 \%$ confidence limit of normal) in the remainder.

Patients with left ventricular hypertrophy had septal or free wall thickening greater than $12 \mathrm{~mm}$ on $M$ mode echocardiography (the upper limit of normal). ${ }^{3}$ Three patients had hypertrophic cardiomyopathy; of the remainder with secondary hypertrophy, seven had aortic stenosis, three arterial hypertension, two had undergone aortic valve replacement, and one repair of coarctation. The patients with rheumatic mitral stenosis had thickened valve leaflets, a reduced diastolic closure rate, and increased mitral diastolic flow velocities detected by continuous wave Doppler. Mitral valve replacement had been undertaken in 18 
Figure 1 Schematic apical four chamber cross sectional view showing the $M$ mode cursor positioned through the posterior aspect of the mitral ring. The cursor angulation was altered slightly to obtain $M$ modes through the left ventricular apex.



1 M mode cursor

patients for rheumatic mitral stenosis and in six for non-rheumatic mitral regurgitation. This was also the preoperative diagnosis in the 10 patients who had undergone mitral repair. All patients with mitral valve disease or replacement had normal left ventricular dimensions and fractional shortening. There was atrial fibrillation in five patients with mitral stenosis, 21 with mitral valve replacement, and five with mitral valve repair. All the remaining patients were in sinus rhythm.
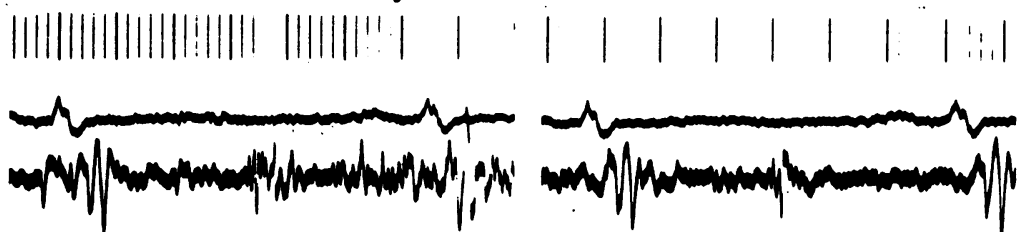

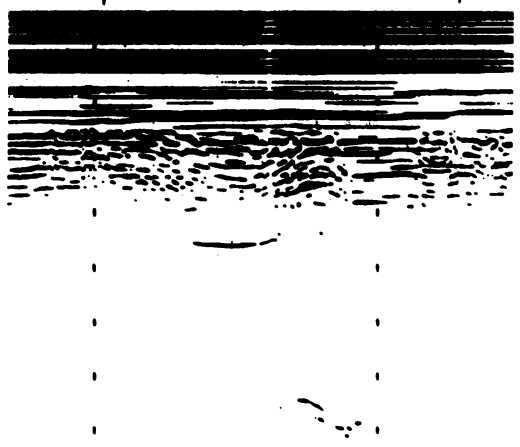

\section{.}
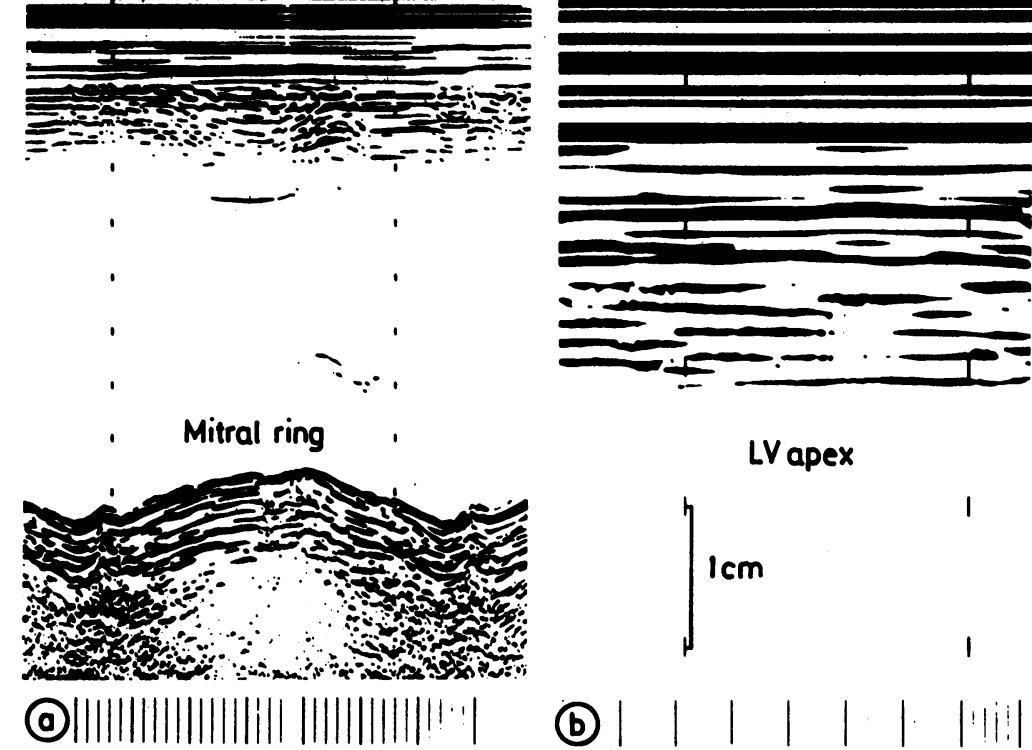

Figure $2 M$ mode echocardiograms of the mitral ring and left ventricular apex in a healthy control. The total amplitude of apical motion was less than $1 \mathrm{~mm}$, indicating that changes in the position of the mitral ring reflect changes in left ventricular long axis dimension.
METHODS

$M$ mode and cross sectional echocardiograms were obtained with the patient lying in the left lateral position with an $860 \mathrm{C}$ Imager with a 3.5 $\mathrm{MHz}$ mechanical transducer. $\mathrm{M}$ mode echocardiograms were recorded on a Honeywell (Ecoline 22) strip chart recorder at a paper speed of $100 \mathrm{~mm} / \mathrm{s}$ with simultaneous electrocardiogram and phonocardiogram. Standard $M$ mode echocardiograms of the left ventricular minor axis were recorded just below the level of the mitral valve leaflets. Cross sectional guided $M$ mode echocardiograms of the left ventricular long axis were then obtained after longitudinal placement of an $M$ mode cursor through the lateral aspect of the mitral valve ring, visualised on an apical four chamber cross sectional view (fig 1). Motion of the apex was recorded from the same position. Since the amplitude of movement was always less than $1 \mathrm{~mm}$ and since it showed no consistent relation to the electrocardiogram (fig 2), we disregarded it, and took overall shortening or lengthening of the long axis of the left ventricle as being reflected in corresponding motion of the mitral ring.

Echocardiograms were matched for the heart rate; the onset of the $Q$ wave was used to time early systolic events and the first high frequency component of the aortic closure sound $\left(\mathrm{A}_{2}\right)$ was used to time early diastolic events. In the patients with atrial fibrillation $R R$ intervals were matched to within $100 \mathrm{~ms}$. The echocardiograms were digitised ${ }^{4}$ and the following measurements were made as the mean value of three beats in each patient.

(a) Fractional shortening, the peak rate of fall of minor axis, and the peak rate of minor axis fibre shortening measured from the standard minor axis echocardiogram (calculated as the peak rate of minor axis change during systole normalised for end diastolic dimension).

(b) Time intervals from the $Q$ wave to the onset of minor axis shortening and to the onset of long axis shortening (ms).

(c) Time intervals from the $Q$ wave to the minimum minor axis and the minimum long axis (ms).

(d) The extent of minor and long axis change during the initial $100 \mathrm{~ms}$ from the $Q$ wave (cm). Outward wall motion starting before the $Q$ wave was considered to be the result of ventricular filling by atrial contraction and was excluded.

(e) The total amplitude of minor axis epicardial excursion (cm), and the time intervals from the $Q$ wave to peak inward endocardial and epicardial excursion on the minor axis echocardiogram $(\mathrm{cm})$.

(f) Total amplitude of long axis shortening (cm).

(g) Time intervals from $A_{2}$ to the peak rates of minor and long axis increase in early diastole (ms).

Mean values for each measurement obtained in the disease groups were derived and compared with values in healthy controls. Standard deviations were calculated and the significance of differences between means was assessed by $t$ tests. 
Figure 3 Minor (a) and long (b) axis

echocardiograms of $a$ control showing the earlier onset of long axis

shortening, and normal

posterior wall thickening.

Changes in long and mino axes were synchronous at end ejection and in early diastole. The $Q$ wave and the vertical lines indicate the onset of fibre shortening.

Figure 4 Minor (a) and long (b) axis echocardiograms of a patient with coronary disease and reduced fractional shortening. Long axis shortening was delayed beyond minor axis shortening so that the normal increase in the minor axis during isovolumic contraction was lost. Similar disturbances were seen in patients with normal fractional

shortening. The abnormal change in shape caused by incoordinate relaxation was evident as an increase in the minor axis and a reduction in the long axis during a prolonged isovolumic period. $A$ aortic closure sound; MVO, mitral valve opening.
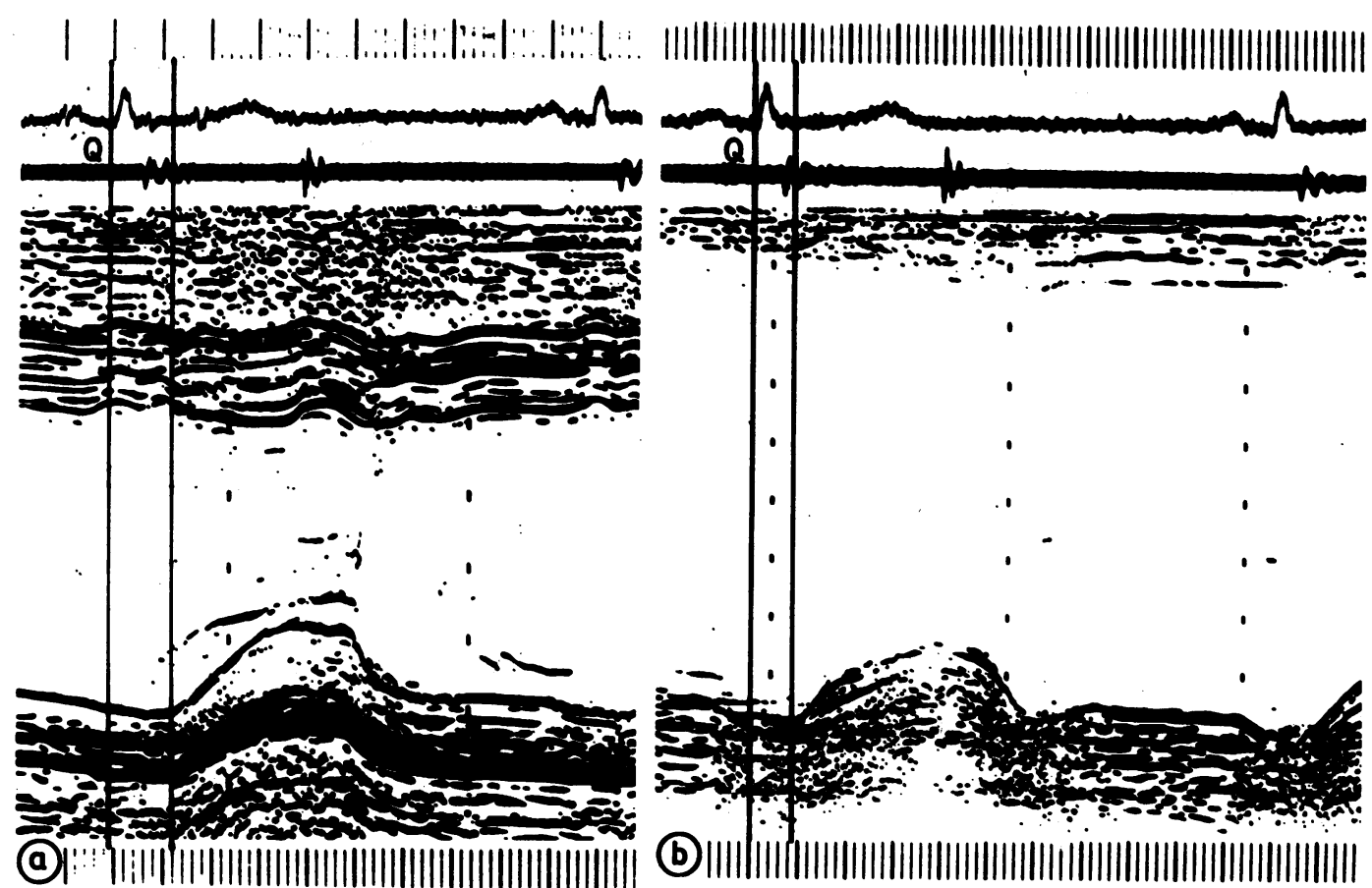

\section{Results}

\section{HEALTHY CONTROLS}

In the controls (fig 3) fractional shortening was $33(5) \%$, peak rate of dimension change was $9(2.9) \mathrm{cm} / \mathrm{s}$, and peak velocity of minor axis fibre shortening was $2.4(0.5) \mathrm{cm} / \mathrm{s}$. There was an initial increase in the long axis during isovolumic contraction of $0.09(0.13) \mathrm{cm}$, significantly less than the corresponding change in the minor axis $(0.25(0.15) \mathrm{cm})(\mathrm{p}<0.05)$, so that the cavity became more spherical. Long axis shortening began 55 (40) ms after the $Q$ wave and preceded shortening of the minor axis by $25(40) \mathrm{ms}$ (p $<0.01)$. Peak shortening of the two axes was effectively synchronous, occurring 410 (55) ms (long) and 430 (50) ms (minor) after the QRS complex $(p=N S)$. However, the timing of epicardial motion differed significantly from that of the endocardium. Peak inward motion of the epicardium consistently preceded that of the minor axis by $50(20) \mathrm{ms}$, so that the epicardium moved outwards and the minor axis continued to shorten during isovolumic relaxation. The overall epicardial excursion was $0.5(0.2) \mathrm{mm}$. The timing of peak rates of long and minor axis lengthening was effectively synchronous in early diastole, occurring $100(25) \mathrm{ms}$ and 90 (30) ms after $\mathrm{A}_{2}$ respectively ( $p=N S$ ).

\section{CORONARY ARTERY DISEASE}

Early systolic events were significantly modified in patients with coronary artery disease (fig 4), whether or not standard measurements of left ventricular function were normal. Minor axis shortening started earlier than normal $(55$ (40) ms $v 80$ (40) ms after the $Q$ wave, $p<0.05$ ), while that of the long axis was delayed (85 (30) ms $v 55$

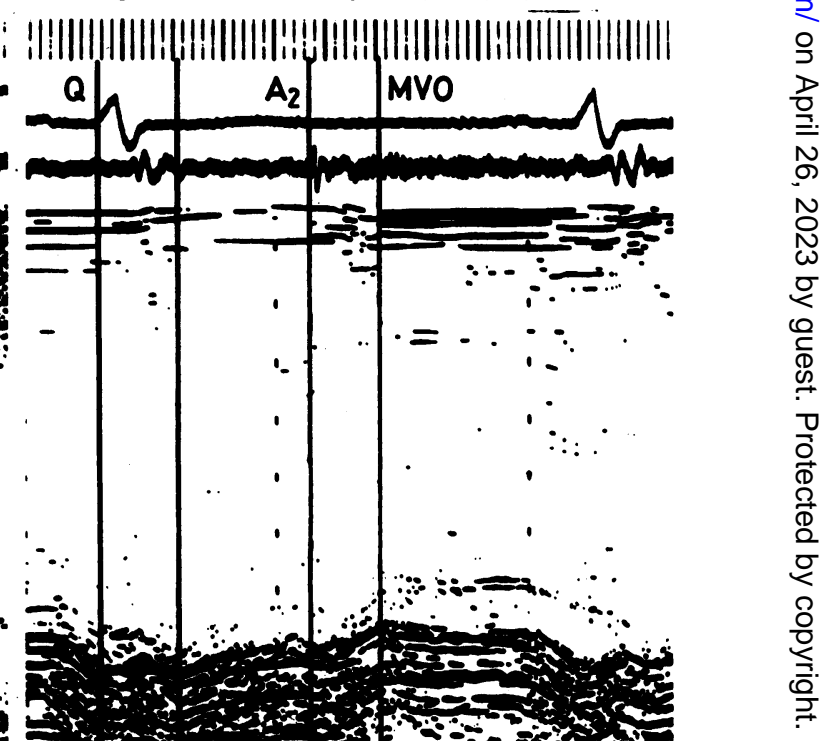




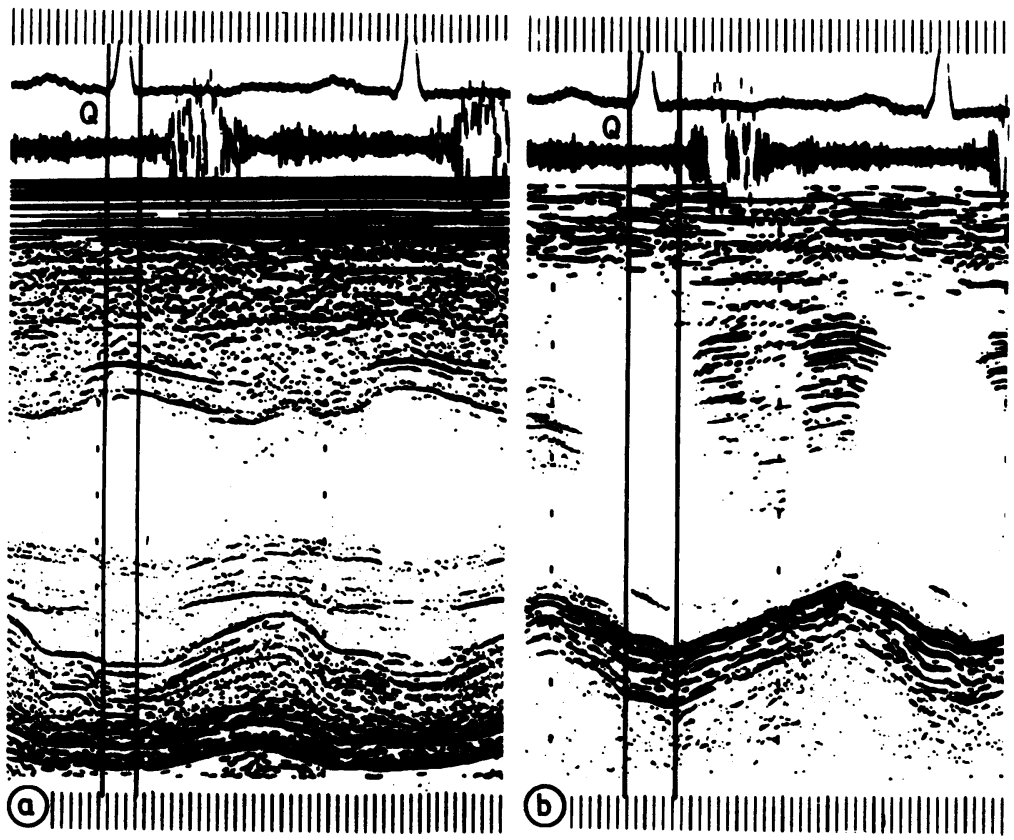

Figure $5 \mathrm{M}$ mode echocardiograms showing delayed long axis (b) shortening with an abnormal shape change during isovolumic contraction despite normal fractional shortening on the minor axis (a) in a patient with aortic stenosis and left ventricular hypertrophy.

(40) ms, p $<0.01)$. The start of minor axis shortening thus preceded that of the long axis, rather than followed it as in the controls. At the same time the pattern during isovolumic contraction changed, with a fall in the extent of minor axis expansion from $0 \cdot 25 \cdot(0 \cdot 15) \mathrm{cm}$ to $0 \cdot 1(0 \cdot 1) \mathrm{cm}(\mathrm{p}<0 \cdot 05)$. The two axes were, however, synchronous at end ejection. The normal asynchrony seen between epicardial and minor axis motion was partly lost because peak epicardial displacement was delayed (difference $30(20) \mathrm{ms}, \mathrm{p}<0.01 v$ control), though the amplitude of epicardial excursion remained normal. During diastole the peak rates of increase of the long and minor axes were synchronous after $A_{2}$.

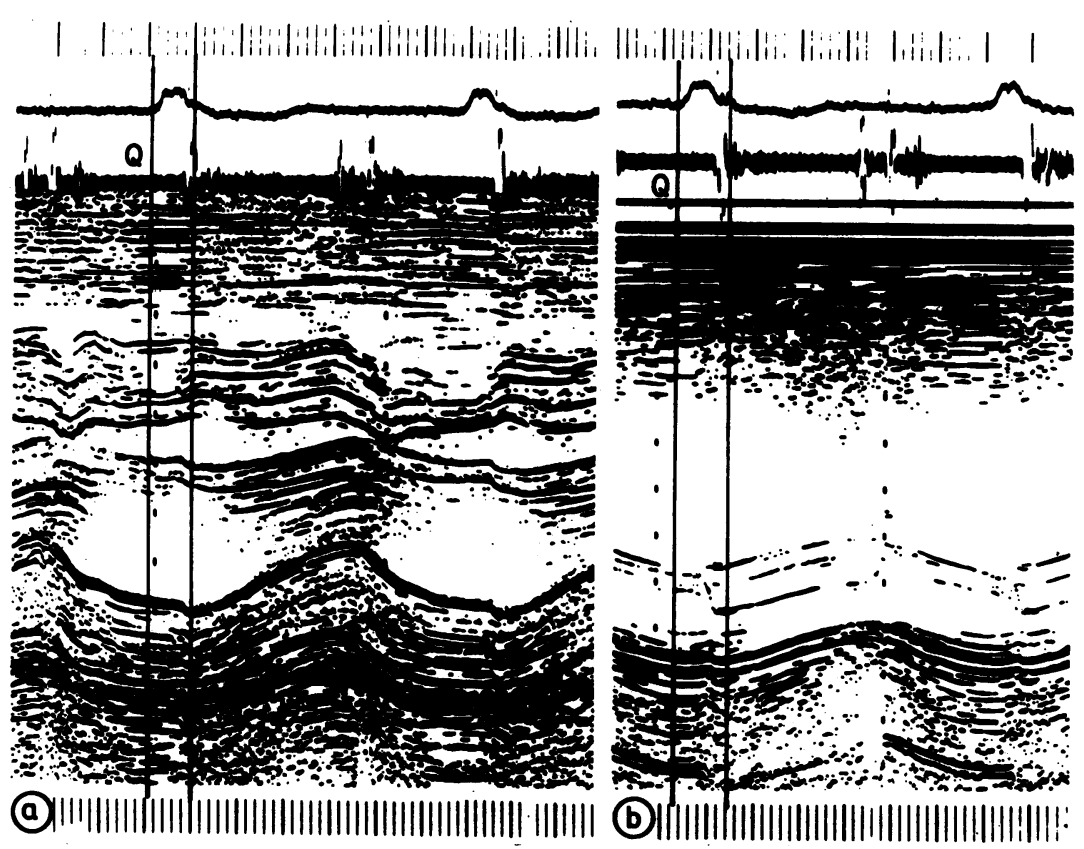

Figure $6 M$ mode echocardiograms showing characteristically delayed and diminished long axis shortening (b) in a patient after mitral valve replacement. Normal minor axis (a) endocardial motion is maintained only by increased epicardial excursion. Peak endocardial and epicardial motions were synchronous in this group.
LEFT VENTRICULAR HYPERTROPHY

Fractional shortening, peak rate of minor axis change, and peak velocity of circumferential fibre shortening were normal in the patients with left ventricular hypertrophy $(30(7) \%$, $8.3(2 \cdot 2) \mathrm{cm} / \mathrm{s}$, and $2.6(0.4) \mathrm{cm} / \mathrm{s}$ respectively, $\mathrm{p}=\mathrm{NS}$ compared with controls). As with coronary artery disease, however, there was considerable initial systolic asynchrony (fig 5). Minor axis shortening again started earlier than normal with respect to the $Q$ wave (60 (30) $v 80(40) \mathrm{ms}, \mathrm{p}<0.05)$ and that of the long axis was delayed (95 (35) $v 55(40) \mathrm{ms,}$ $\mathrm{p}<0.01)$. Short axis expansion during isovolumic contraction was reduced $(0.05$ $(0.1) v 0.25(0.15) \mathrm{cm}, \mathrm{p}<0.01)$ though long axis expansion was unaltered. However, despite their asynchronous onset peak shortening of the long and short axes was synchronous as was early diastolic filling. The extent of long axis shortening was normal. Peak epicardial excursion preceded peak endocardial excursion as in the controls (difference 45 (30) $\mathrm{ms}$ ), and epicardial excursion itself was again normal.

\section{MITRAL STENOSIS}

Standard measures of left ventricular function were also normal in this group (fractional shortening $34(8) \%$, peak rate of dimension change $7.5(3.4) \mathrm{cm} / \mathrm{s}$, and peak velocity of fibre shortening $2.3(0.3) \mathrm{cm} / \mathrm{s})$. However, the onset of long axis shortening was later (105 (35) $v 55$ (40) $\mathrm{ms}, \mathrm{p}<0.01)$ and its amplitude lower $(1.0$ $(0.3) v 1.3(0.2) \mathrm{cm}, \mathrm{p}<0.05)$ in patients with mitral stenosis than in controls. The timing of the onset of minor axis shortening was normal (75 (25) ms after the $Q$ wave, $p=N S$ ) and so shortening of the minor axis preceded that of the long axis. The long axis increased more than the minor axis during isovolumic contraction $(0.4(0.2)$ v $0.15(0.2) \mathrm{cm}, \mathrm{p}<0.01)$. Changes in the long and short axes were synchronous at end systole and during early diastole. Peak minor axis and epicardial shortening almost coincided (difference $20(20) \mathrm{ms}$ ) although the amplitude of the epicardial excursion was normal.

\section{MITRAL VALVE REPLACEMENT}

Time relations were very abnormal after mitral valve replacement (fig 6 ). Long axis shortening was delayed (onset $95(50) v 55(40) \mathrm{ms}, \mathrm{p}<$ $0.01)$ and of reduced amplitude $(0.9(0.4) v 1.3$ $(0.2) \mathrm{cm}, \mathrm{p}<0.01)$ while the amplitude of shortening of the minor axis was normal. The onset of minor axis shortening was much earlier than normal (45 (35) $v 80(40) \mathrm{ms}, \mathrm{p}<0.01)$ without significant increase during isovolumic contraction $(0.05(0.1) v 0.25(0.15) \mathrm{cm}$ normal, $\mathrm{p}<0.01)$. Wall motion was strikingly asynchronous at end systole, with peak minor axis shortening preceding that of the long axis by 70 (15) $\mathrm{ms}(\mathrm{p}<0.01)$, and during early diastole (peak lengthening rate minor axis $105(25) \mathrm{ms} v$ 195 (45) $\mathrm{ms}$ after $A_{2}, p<0.01$ ). There was also a loss of the normal asynchrony across the ventricular wall, the difference between peak epicardial and minor axis shortening being only $10(30) \mathrm{ms}(\mathrm{p}<0.01)$. The normal amplitude of 
minor axis reduction was maintained by an increased amplitude of epicardial excursion $(0.9(0.3) v 0.5(0.2) \mathrm{cm}$ normal, $\mathrm{p}<0.01)$. These changes were again present despite normal fractional shortening, peak rate of dimension change, and peak velocity of fibre shortening $(31(6) \%, 7 \cdot 8(2 \cdot 2) \mathrm{cm} / \mathrm{s}$, and $2 \cdot 4(0 \cdot 4) \mathrm{cm} / \mathrm{s}$ respectively).

\section{MITRAL VALVE REPAIR}

In patients after mitral repair the onset and amplitude of long axis shortening were not significantly different from those in controls (onset $90(70) v 60(40) \mathrm{ms}, \mathrm{p}=\mathrm{NS}$ ) and events at end ejection and in early diastole were synchronous. The only disturbance in these patients was a slight increase in epicardial excursion $(0.7(0.2) v 0.5(0.2) \mathrm{cm}, \mathrm{p}<0.05)$. Standard measures of left ventricular function were normal (fractional shortening $28(6 \%)$, peak rate of dimension change $8.4(1.8) \mathrm{cm} / \mathrm{s}$, and peak velocity of fibre shortening $2(0.5)$ $\mathrm{cm} / \mathrm{s}$ ).

\section{Discussion}

The way in which the functions of the longitudinally and circumferentially orientated myocardial fibres are interrelated to give a coordinated left ventricular contraction has been the subject of anatomical and physiological study for many decades. Straub ${ }^{5}$ and subsequently Rushmer ${ }^{6}$ showed that the longitudinal fibres started to shorten before those of the minor axis so that the cavity became more spherical-a sequence of events subsequently shown by Rankin et al to be related to the exact animal preparation used. Another aspect of these interrelations has been investigated more recently-the extent to which longitudinal shortening contributes to wall thickening and thus to minor axis changes. ${ }^{89}$ In spite of these studies, however, there has been little work on changes in the human long axis, and particularly on the effect of left ventricular disease. Because the apex of the left ventricle does not move significantly with respect to the transducer, long axis changes are reflected in movements of the mitral valve ring. Thus we have been able to study the relations between shortening of the long and minor axes of the left ventricle by simple echocardiographic techniques.

Our study confirmed previous experimental work by showing consistent differences in the timing and extent of long and minor axis shortening in controls. We showed the characteristic change in cavity shape during isovolumic contraction that occurs because shortening of the minor axis lags slightly behind that of the long axis. We also confirmed the presence of asynchrony across the posterior wall with the peak inward motion of the epicardium preceding that of the endocardium. This means that maximum long axis shortening, though synchronous with peak inward endocardial motion, is strikingly delayed with respect to that of the epicardium. These findings provide further examples of the regional non-uniformity of function that we and others have explored in the normal left ventricle. ${ }^{10-12}$
There were characteristic changes in these relations in patients with heart disease. With groups of widely differing aetiology, the normal sequence of early systolic events was consistently altered. Shortening of the minor axis started prematurely, longitudinal shortening was delayed, and changes in cavity shape during isovolumic contraction were abnormal. To test the hypothesis that these changes were the direct result of impaired function of the longitudinal fibres we studied patients after mitral valve replacement because in this operation the papillary muscles, a component of the longitudinally directed fibres, are cut. We found that wall motion was particularly abnormal in these patients. Not only was the normal early systolic sequence of events disrupted but also the extent of longitudinal shortening was reduced and the abnormal asynchrony between long and minor axes persisted throughout ejection and into early diastole. These changes were not a specific effect of cardiopulmonary bypass because they did not occur to the same extent after mitral valve repair, nor could they be attributed to a minor degree of mitral valve obstruction by the prosthesis because they were present to only a limited extent in cases of well developed mitral stenosis. We therefore attribute them directly to loss of a component of the longitudinally arranged muscle. Abnormal systolic performance has been lessened and survival improved after mitral valve surgery when the chordae tendineae were preserved $^{13-16}$; however, longitudinal left ventricular function has not been examined specifically.

Our results also showed that a normal left ventricular shortening fraction after mitral valve replacement is maintained only by a very abnormal mechanism. The main basis of inward motion of the endocardium was changed: instead of being the effect of wall thickening as in the controls, it was the result of increased amplitude of motion of the epicardium. This fundamental change is exactly that predicted from anatomical studies ${ }^{8917}$ and is compatible with loss of the role of longitudinal fibre shortening in bringing about myocardial thickening, which determines the normal dynamics of the minor axis.

The results in patients with ischaemic heart disease were also instructive. Again abnormal long axis dynamics were associated with complex disturbances of ventricular function. The changes during isovolumic contraction caused by delayed longitudinal shortening resembled those seen in patients after mitral valve replacement. Epicardial amplitude remained normal but the normal lag in peak endocardial motion in relation to that of the epicardium was lost, particularly in patients in whom there was a change in cavity shape during diastole. This impairment of left ventricular longitudinal function in coronary disease is likely to have a basis in fibre architecture. Subepicardial and subendocardial fibre layers are longitudinally orientated whereas the middle layer is circumferentially arranged. Non-uniformity of fibre orientation was thus matched by a nonuniform susceptibility of the various layers to 
injury. Necropsy studies in patients with coronary disease show that the subendocardial fibres are more susceptible to diffuse ischaemic damage. ${ }^{18}$ Subendocardial dysfunction is thus likely to affect selectively the longitudinally directed fibres and so to manifest itself as abnormal long axis shortening. Myocardial hypertrophy alone will also be associated with abnormal long axis dynamics if diffuse subendocardial damage reflects a relatively high risk of anoxia. Indeed we consistently found pronounced early systolic asynchrony and disorganised posterior wall thickening in patients with left ventricular hypertrophy, abnormalities that closely resemble those in patients with known coronary disease. They are the characteristic changes one would expect in the presence of disturbed shortening of longitudinal fibres.

Despite the obvious importance of coordinate contraction of all muscle layers in maintaining optimal ventricular function, the relative contributions of differently orientated myocardial fibres have been little studied in humans. Echocardiographic assessment of long and short axis changes allows an appreciation of the characteristic relations between the two. Their exact biological significance has still to be determined but we feel sure that it is closely related to the processes of energy transfer from the myocardium to the circulation. It also seems clear that loss of these close time relations may be an early sign of ventricular disease, occurring at a stage when fractional shortening is still maintained. There is still controversy about whether systolic left ventricular function is depressed in patients with left ventricular hypertrophy when cavity size and ejection fraction remain normal. ${ }^{19}$ So it may be significant that we were consistently able to show systolic abnormalities in such patients, though they seemed to be confined to the function of the longitudinally directed fibres. Our findings may also shed light on the nature of the left ventricular disease after mitral valve replacement. Such disease is a major cause of long term morbidity and mortality after mitral valve replacement, although orthodox measures of left ventricular function may be normal in the early postoperative period. ${ }^{20}$ It seems that there is a strong rational basis for any attempt to preserve the integrity of the mitral apparatus during operation. Finally, we regard our findings as a clinical vindication of the remarkable conclusions of Sir Arthur Keith, propounded over 70 years ago. ${ }^{21} \mathrm{He}$ commented on the interrelations between the "massive circular expelling coat", which he regarded as the agonist, and the longitudinal fibres (the antagonist) which supported them and prevented abnormal changes in cavity shape. Such changes, particularly when they occur in the two isovolumic periods, have been shown to cause striking loss of mechanical efficiency of ventricular pump function. ${ }^{22}$ We feel that, taken together, these results again raise the question as to the best method of assessing left ventricular systolic function. As it becomes apparent that normal endocardial motion may be brought about by abnormal means we question whether attempts to summarise left ventricular systolic function as a single entity such as contractility or end systolic elastance are limiting. Rather, we sug gest that the aim should be to assess the function of each of the anatomical components within the ventricles individually and to determine how these are integrated to give rise to the final pattern of wall motion.

C J H J is a British Heart Foundation Junior Research Fellow.

1 Greenbaum RA, Ho SY, Gibson DG, Becker AE, Anderson RH. Left ventricular fibre architecture in man. Br Heart 1981;45:248-63.

2 Streeter DD, Spotnitz HM, Patel DP, Ross J Jr, Sonnenblick EH. Fibre orientation in the canine left ventricle during diastole and systole. Circ Res 1969;24:339-47.

3 Gibson DG, Traill TA, Hall RJC, Brown DJ. Echocar diographic features of secondary left ventricular hypertrophy. Br Heart J 1979;41:54-9.

4 Gibson DG, Brown DJ. Continuous assessment of left ventricular shape in man. Br Heart J 1975;37:904-10.

5 Straub $\mathrm{H}$. The diastolic filling of the mammalian heart. Physiol (Lond) 1910; 40:378-88.

6 Rushmer RF. The initial phase of ventricular systole: asynchronous contraction. Am J Physiol 1956;184:188-94.

Rankin JS, McHale PA, Arentzen CE, Ling D, Greenfield JC, Anderson RW. The three-dimensional geometry of the left ventricle in the conscious dog. Circ Res 1976;39:304-13.

8 Dumesnil JG, Shoucri RM, Laurenceau J-L, Turcot J. A mathematical model of the dynamic geometry of the intact left ventricle and its application to clinical data. Circulation 1979;59:1024-34

9 Greenbaum RA, Gibson DG. Myocardial structure of the left ventricle in health and disease. In: Hamer J, Rowlands DJ, eds. Recent advances in cardiology 8. Edinburgh Churchill Livingstone, 1980:207-31.

10 Shapiro E, Marier DL, St John Sutton MG, Gibson DG Regional non-uniformity of wall dynamics in normal left ventricle. Br Heart J 1981;45:264-70.

11 Haendchen RV, Wyatt HL, Maurer G, et al. Quantitation of regional cardiac function by two dimensional echocardiography. 1. Parterns of contraction in the normal left ventricle. Circulation 1983;67:1234-45.

12 Hammermeister KE, Gibson DG, Hughes D, and paricipant in Veterane Adminietration Cooperative study ticipants in Vecerans Adminisuracion Cooperative Study (he timing and extent of left vent (6.226-35.

13 Lillehei CW, Levy MJ, Bonnabeau RC. Mitmal valve replacement with preservation of papillary muscles and chordae tendineac. J Thorac Cardiovase Surg 1964;47:532-43.

14 David TE, Burns RJ, Bacchus CM, Druck MN. Mitral valve replacement for mitral regurgitation with and withou preservation of chordae tendineae $J$ Thorac Cardiovase Surg 1984;88:718-25.

15 Bonchek LI, Siegel R, Olinger GN, Keelan MH, Tresch DT. Left ventricular function is better after mitral valve repair than after valve replacement [Abstract]. Am Cardiol 1982;49:922.

16 Hansen DE, Cahill PD, DeCampli WM, et al. Valvularventricular interaction: importance of the mitral apparatus in canine left ventricular systolic performance. Circulation 1986;73:1310-20

17 Armour JA, Randall WC. Structural basis for cardiac function. Am J Physiol 1970;218:1517-23.

18 Davies MJ, Roberteon WB. Diseases of the coronary arteries. In: Pomerance A, Davies MJ, eds. The pathology arteries. In: Pomerance A, Davies MJ, eds. The pathology of the heart.

19 Pouleur H, Rousseau MF, van Eyll C, Brasseur LA, Charlier AA. Force-velocity-length relations in hypertrophic cardiomyopathy: evidence of normal or depressed myocardial contractility. Am J Cardiol 1983;52:813-7.

20 Kennedy JW, Doces JG, Stewart DK. Left ventricular function before and following surgical treatment of mitral valve disease. Am Heart $J$ 1979;97:592-8.

21 Keith $\mathrm{A}$. The functional anatomy of the heart. $\mathrm{Br}$ Med 1918:1:361-3.

22 Gibson DG, Brown DJ. Assessment of left ventricular systolic function in man from simultaneous echocar1976;38:8-17. 